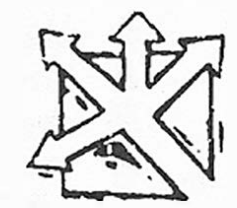

Educación Superior

\title{
CAMBIAR PARADIGMAS: UNA EXPERIENCIA EN EDUCACIÓN SUPERIOR
}

\author{
Vanesa Alvarado Chacón' \\ María de los Ángeles Montero Dien² \\ Gisella Vargas Chinchilla \\ Silvia Chacón Ramírest
}



1 Licenciada en Educación Preescolar. Investigadora. Maestra Colegio Nuestra Señora del Pilar.

2 Licenciada en Educación Preescolar. Investigadora.

3 Licenciada en Educación Preescolar. Educadora Ambiental Fundaos.

4 Licenciada en Educación Preescolar. Máster en Planificación Curricular. Profesora de la Universidad de Costa Rica. Directora de la investigación. 


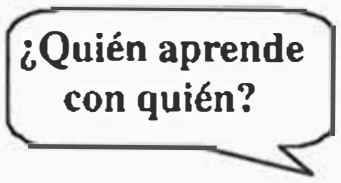

Lo regular en el desarrollo de ambientes educativos, lo constituye la utilización de un modelo tradicional o vertical, donde docentes cumplen un papel de informantes, transmisores o guías y los estudiantes un papel de receptores del conocimiento.

Quién podría suponer la existencia de un proceso de autoformación, donde docentes y estudiantes comparten dentro de una comunidad de aprendizaje, en busca de una meta en común. Con la particularidad de que el papel mediador es desempeñado por estudiantes, lo cual se constituye en un cambio de paradigma.

Pues bien, esta es la experiencia que el presente artículo describe y que se ubica en el contexto de la Educación Superior, específicamente en la carrera de Educación Preescolar de la Universidad de Costa Rica.

El proceso de autoformación en mención se desarrolló al estilo de una investigación-acción, durante más de un año. En esta experiencia el objetivo principal fue promover que el personal docente, que ejecuta el plan de estudio de Educación Preescolar de la Universidad de Costa Rica, se constituyera en ente multiplicador del eje transversal de Desarrollo Sostenible, dentro del desarrollo de dicho plan de estudio.

Ahora bien, para alcanzar su objetivo, las investigadoras utilizaron no sólo sus conocimientos como educadoras, sino también sus actitudes de responsabilidad, profundización del conocimiento, sus aptitudes para el trabajo en equipo, así como su fidelidad al principio de valorización del ser humano y del ambiente.

Este proceso dio inicio en el mes de julio del 2001, cuando VanesaAlvarado, Marielos Montero, Meybol Rodríguez, Milagro Vargas y Giselia Vargas, todas estudiantes de la carrera de Educación Preescolar, decidieron conformar un equipo de investigación, con el fiı me propósito de realizar su Trabajo Final de Graduación, para optar al grado de Licenciadas en Educación Preescolar de la Universidad de Costa Rica.

Conscientes del reto que implicaba, en el ámbito personal, profesional e investigativo, propusieron ante la coordinación de la Sección de Educación 
This is a research-acsion rype of experience in higher educarion lived by a "learn. ing commmnity" conformed by teachers and students whose aim is to become fostering agents for sustesincable development. The cxperience was carriced ont as part of the plan for seacher rasuing in Prexchool Edncusion.

This paper is the eurthor's Final Gradu. arion Papes: a recgurement to obnain a Licensiase degree in Preschool Educution as she University of Costa Rica. The research began with a diagnosis that would lead ro she implementation of an action plan ehat was oryenrized and carricd ont joindly by she stu. deats and sheir peachers. The project finished with the parsicipants' acceptunce of some "environmental comminments".
Preescolar; el tópico de "Educación Ambiental" como árcal de intcres. La respuesta de la sección, cn la persona de la profesora Silvia Chacón fue inmediata, quicn se interesó en la propuesta y apoyó la iniciativa a través de la orientación del grupo en la definición del temay cl cnfoquc investigativo de abordajc.

Posteriormentc, sc solicitó a la Dra. Claudia Charpenticr y a la Licda. Andrea Anfossi, su colaboración como ascsoras, la primera por su experiencia y formación en cl campo de la Educación Ambiental, así como por su aporte investigativo

en el campo de la biología dentro de la Universidad Nacional; y la segunda por su formación en educación preescolar; su reconocida trayectoria en el ámbito de la Informática Educativa dentro de la Fundación Omar Dengo, así como por su amplia expericncia en el campo de las capacitaciones y la incorporación de nuevas tecnologías a la coducación.

Para cl sustento tcórico se tomaron como base las ideas propuestas por:

- Kathcrine Emmons (1997), con su Modelo de acción ambicntal positivo.

- Michael Caduto (1985), y su modclo de valores ambientalcs instrumentales y terminales.

- La Comisión Interuniversitaria (2002) y sus ideas sobrc el cmpoderamiento de conocimientos, actitudes, valores y comportamientos ambientales.

Es así que para explicar csta experiencia de "cambio de paradigma", sc responde a las siguientes interrogantcs:

- ¿Qué se cnticnde por proceso de autoformación?

- ¿Para qué y para quiéncs cs cl proceso de autoformación?

- ¿Cómo sc llevó a cabo cl proceso de autoformación?

- ¿Qué gencró este proceso de autoformación?

- ¿Qué se concluyc al finalizar el proceso de autoformación? 
¿Qué se entiende por proceso de autoformación?

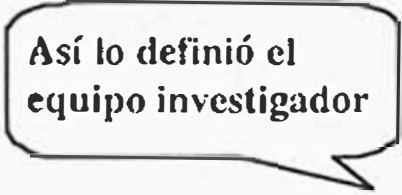

Etimológicamentc cl término autoformación significa formación de sí mismo.

Desde una perspectiva más amplia, la autoformación se entiende como un proceso planificado, que da respuesta a una o varias necesidades, para la aprehensión, en la persona, grupo o institución, de conocimientos. habilidades o valores, o las tres, de los cuales carecía, mediantc un proceso colaborativo que busca la complementariedad e intercambio de experiencias y recursos en un ambiente de reflexión, construcción y rcconstrucción de conocimientos y propuestas pedagógicals concrelas (Alvarado y otras, 2002).

Por su parte, durante la experiencial las profesoras y los profesores conceptualizarion la "comunidad de aprendizaje" comoaquella dondesc incorporan los clementos naturales. personas pensantes, emociones, aprendizaje, dentro de un proceso colaborativo.



\section{¿Para qué y para quiénes se dirige el proceso de autoformación?}

Para qué...

Para buscar un efecto multiplicador que trascendicra a cducadores, jóvenes estudiantes, niños (as), mediante la incorporación en cl quehacer docentc, de una dimensión ambiental, tal y como se visualiza en el siguiente csquema:

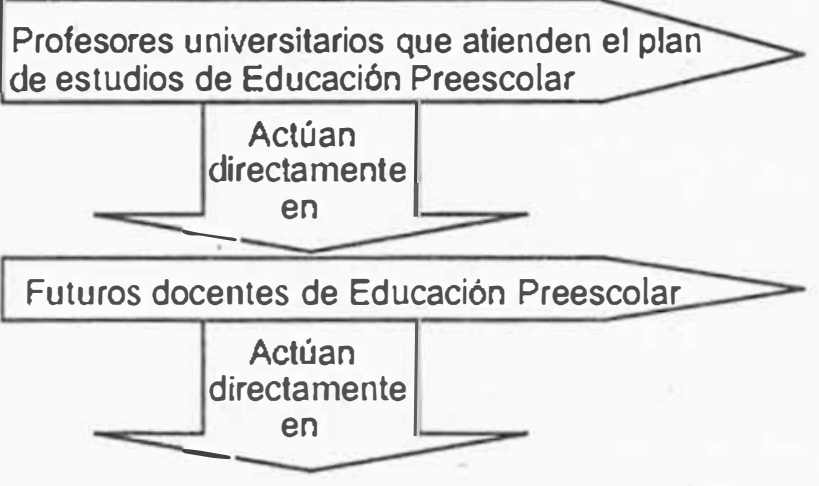

Niños y niñas en edad preescolar y sus familias



Sus familias, compañeros de trabajo y personas con quienes comparten 


\section{Para quiénes...}

- Para las profesoras y los profesores que atienden el plan de estudios de Bachillerato y Licenciatura de Educación Preescolar.

- Para los especialistas que compartieron en algún momento el proceso de autoformación.

De esta manera, el sujeto y el objelo interactuaron, siendo inseparables, existiendo una cstable relación entre ambos con implicaciones para la investigación (Dobles y otras, 1996).

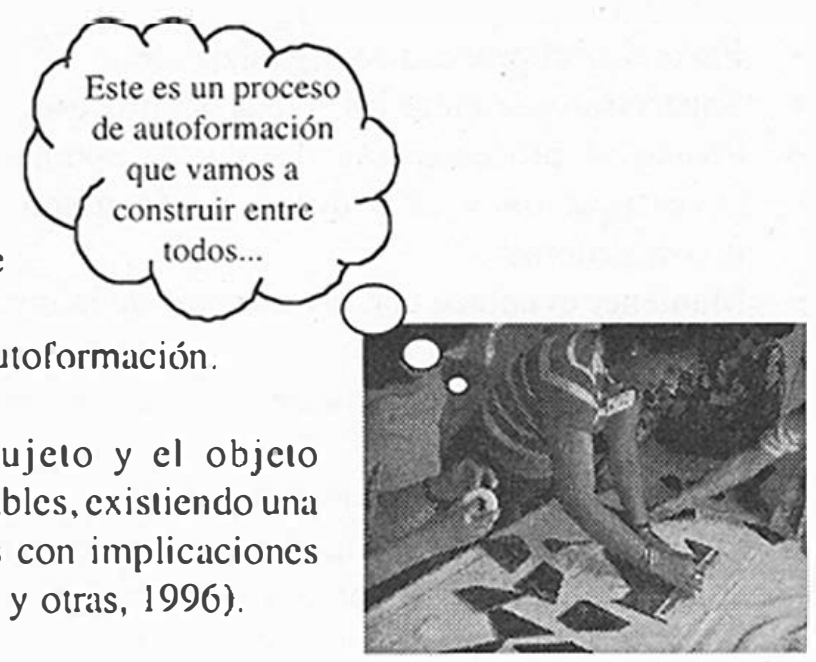

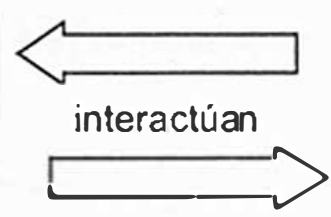

Objeto:

Autoformación en

Educación

Ambiental
La comunidad de aprendizaje funciones. desempeñó ciertos papeles y

El papel de las investigadoras consistió $\mathrm{en}$ :

- Ser mediadoras y faciliadoras cle procesos: interacción con los sujelos paral oblener información, convivio con su realidad. promoción de la reflexión y la construcción de propuestas.

- Ser planificiadoras de las estratcgias y los recursos que mejor alcndieran las necesidiales de autoformación.

- Ser sistemalizadoras del proceso propio de la investigación. en sus dimensiones evaluativa y de autoformación, asi como del proceso metaliognoscitivo propio del equipo, que llevó a la concientización de su formacicin como investigadoras. 
Por su parte la directora de la investigación tuvo a su cargo las siguientes luncioncs:

- Coordinar al cquipo investigador.

- Favorecer el proceso de organización.

- Supcrvisar y orientar las clapas del proceso.

- Promover procesos reflexivos y de aprendizaje, en torno al proceso de investigación y al proceso de formación de las csludiantes como investigadoras.

- Mantener cl cnlace con las ascsoras de la investigación.

Mientras tanto las ascsoras tuvicron la responsabilidad dc:

- Conocer cl proyecto de investigación.

- Definir al cquipo investigador su foco de atención en cuanto al trabajo.

- Definir junto con la dircctora los mecanismos de comunicación.

- Analizar, reflexionar y sugerir junto con cl cquipo investigador.

- Mantener informadas a las investigadoras, en forma oportuna, de sus apreciaciones y sugcrencias mediante informes orales, escritos y otras formas de comunicación.

Asimismo, las profesoras y los profesores participantes cumplicron las siguicntes funcioncs:

- Palticiparactivamente de las sesiones presenciales y no presencialcs, intcractuando con los compañeros $\mathrm{c}$ investigadoras, mediantc un proceso reflexivo.

- Sugcrir temáticas, cstratcgias c idcas durante cl proceso de autoformación.

- Construir conceptos, acciones didácticas.

- Cumplir con los compromisos adquiridos concl proceso de autoformación, asistencia a las diferentes sesiones, completar bitácoris personales y presentar cl portafolio.

¿Cómo se llevó a cabo el proceso de autoformación?

Se utilizó un referente conceptual
El referente tcórico consideró tres modelos, de los cualcs se presenta un extracto a continuación:

1. Los valores ambientales propucstos por Michacl Caduto (1985). Scgún cstc autor, los valores son una preferencia fucitc y duradera que un individuo o grupo tienc para un objcto, conducta o modo de vida. Los clasifica en dos 
grupos: los valores instrumentales y los valores finales o terminales. Para Caduto la formación de valorcs constituye un proceso social determinado por la influencia de la sociedad en el individuo. Dentro de los principales cntes formadores se encuentran la familià, los profesores, las autoridades religiosas, los compañeros, el gobierno, cl trabajo, los medios de comunicación, la literatura y las leyes.

2. La acción ambiental positiva de Katherine Emmons (1997), quien considera tres elementos básicos
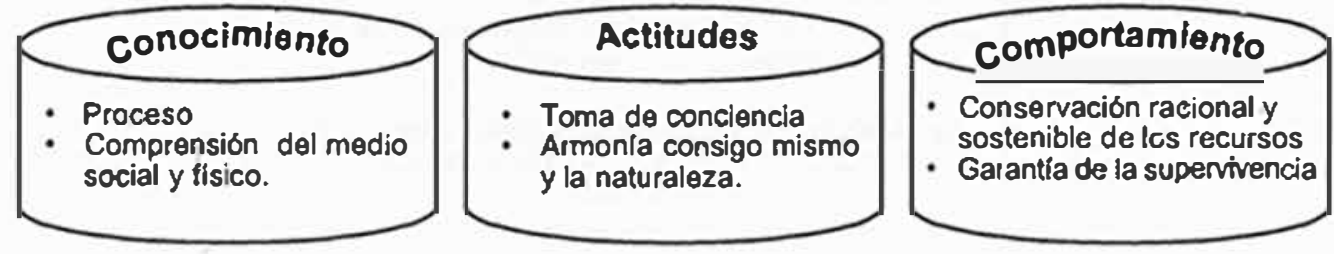

Una vez que la persona logra integrar cstos clementos, entonces alcanza una actitud de "cmpodcramicnto y pertenencia" que le permite llegar a una "acción ambiental positiva". Según Emmons, en este modelo las relaciones son interactivas, dinámicas, detcrminadas por los participantes y las situaciones sociales dentro de las cuales opcran. Esta dinámica tiene la intención de alcanzar un resultado pequeño o grande.

3. El aportc de la Comisión Interuniversitaria de Educación Ambiental del CONARE (Mata y otras, 2002).

Esta comisión amplia cada uno de los aspectos involucrados en cl modelo de Katherinc Emmons, y agrega las "crecncias de los involucrados", como un clemento de influcncia. La cuales las define como aquellos "aspectos evaluativos, atributivos. informativos, culturales o individuales, que predisponen una acción o comportamiento" (Obregón , 1996: 6. citado por Mata y otras, 2002):

También considera que las actitudes y valores influyen en los conceptos y habilidades que se tengan para lograr un "empoderamicnto"

De csta manera cl cquipo de investigación decidió desartollar toda la propuesta tomando en cuenta los conocimientos ambientales, las actitudes y los valores, los comportamicntos ambientales positivos, incluycndo las habilidades y destrezas, para desarrollar una posición llamada Comportamiento Ambiental Reflexivo. 
Se utiłizó además un paradigma investigativo

La investigación se desarrolla dentro del paradigma cualitativo, pues constituye un proceso de reflexión, conceptualización y acción, en un procedimiento sistemático y riguroso (Brenes, 1992).

Las principales caracteristicas de este paradigma, según Pérez (1990), se resumen en el siguiente esquema, donde se observa su vínculo con la presente investigación:

Tiene una visión hollstica: busca comprender la conducta humana desde el propio marco de referencia de quien aclúa.

Los participantes fueron docentes universitarios, cuyo proceso do deserrollo fue tanto profosional como personal.

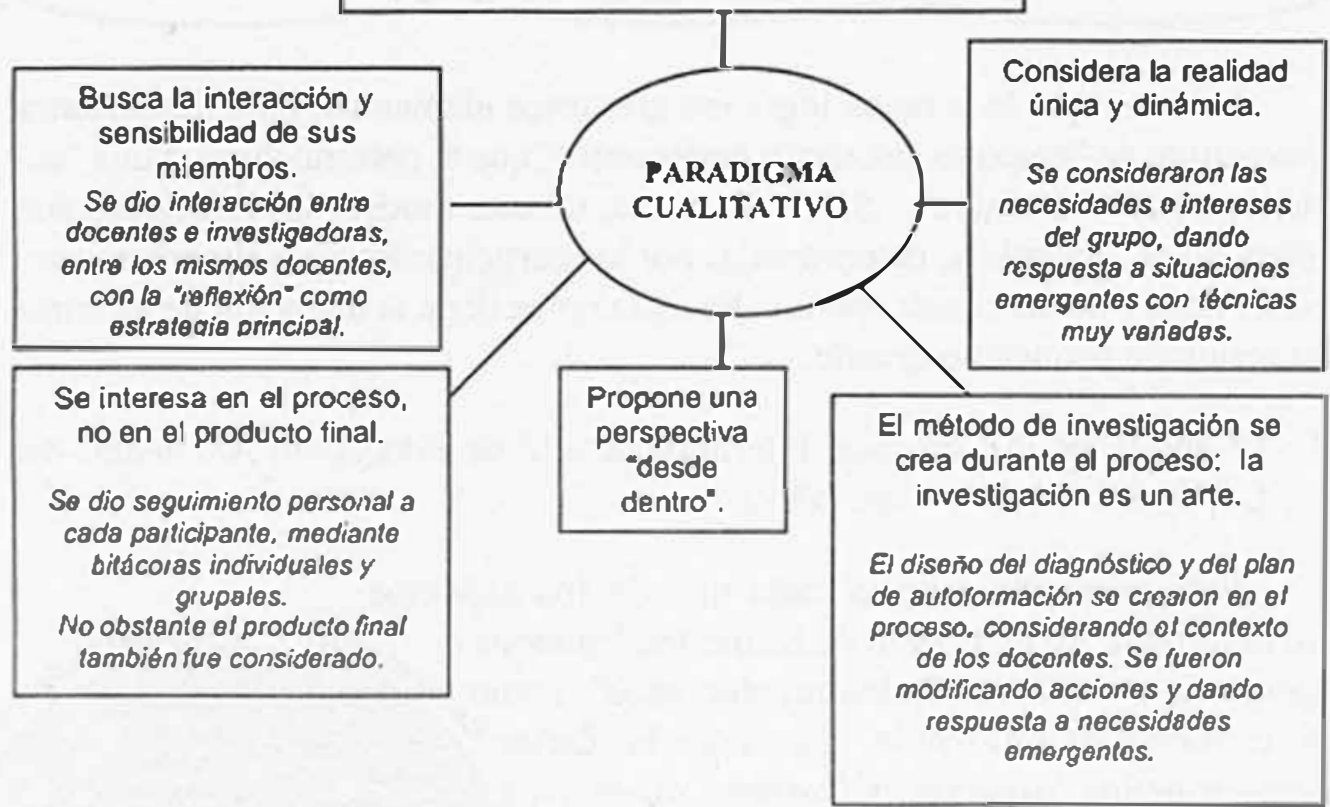

Sc utilizó un estilo de investigación
Dentro del paradigma, se utilizó la investigación-acción, la cual se caracteriza por:

- Tener un carácter participativo.

- Promover un impulso democrático.

- Provocar compromiso con el proceso, participación y actliación en el mismo.

- Relacionar la formación documental, práctica e investigadora.

- Buscar la cooperación y la automodificación comunicativa. 
- Implicar la autorieflexión en una metodología participativa y colaborativa en la que los investigadores se involucran, dando lugar a la planificación, acción, observación y revisiones continuas para reflexionar constantemente.

- Generar una aclividad por grupos o comunidades para contribuir al cambio social.

- Posecr una metodología amplia y flexible.

Por sus características esıc estudio se enmarca dentro de la investigación-acción crírica encuncipatorica, la cual Colás (1994) describe como la que pretende conectar expresamente la acción de los practicantes con las condiciones sociocontextuales en las que se desenvuclve, pretendiendo provocar cambios no solo individuales, sino organizativos y socia-


les. Este tipo de investigación supone un grado de participación alto, ya que se manifiesta en tres niveles observables en la autoformación.

\begin{tabular}{|c|c|}
\hline $\begin{array}{c}\text { Niveles } \\
\text { (según Barbicr, citiclo } \\
\text { por Colás, 1994) }\end{array}$ & Manifestaciones en la autoformación \\
\hline Psicoafectivo & $\begin{array}{l}\text { Las y los profesores expresaron por medios } \\
\text { escritos y orales sus sentimientos, actitudes y } \\
\text { valores, respecto al proceso mismo y la rela- } \\
\text { ción con el ambiente. Además, evaluaron sus } \\
\text { acciones, conocimientos y actitudes median- } \\
\text { le ejercicios de sensibilización. También com- } \\
\text { prendieron y conocieron las runciones y } \\
\text { habilidades de sus compañeros. }\end{array}$ \\
\hline Histórico-existencial & $\begin{array}{l}\text { Tanto docentes como investigadoras, compar- } \\
\text { lieron una historia y un contexto social: la } \\
\text { Sección de Educación Preescolar y el plan de } \\
\text { estudios de Educación Preescolar. }\end{array}$ \\
\hline Estructura!-profesiona! & $\begin{array}{l}\text { Tanto docentes como investigadoras, reflexio- } \\
\text { naron hasta tomar conciencia de la propia si- } \\
\text { tuación personal, profesional e investigativa, } \\
\text { asi como su compromiso con el ambiente. } \\
\text { Se vivió un compromiso personal y grupal. }\end{array}$ \\
\hline
\end{tabular}


Se utilizaron cslralegias

Para lograr la investigación-acción crítical cmancipaloria fucron neccsarios dos procesos: la reflexión y la sistematización. La reflcxión sc dió posterior o antcrior a la ejccución, planificación y observación del proccso. Lal comprensión interpretiltival se logró por medio del diálogo sin restriccioncs con las y los participantes del proyecto. El discurso y la reflexión sobre cl mismo son axiomáticos.

Por su parte, la sistcmatización fue un proceso, colcctivo en este calso, donde se trató de reconstruir críticamente la príctical del proyecto, a través de là identificación y ordenamiento de los clementos y vivencias que conformaron cl desiarrollo del mismo. Se empleó un sistema formal $\mathrm{c}$ intencionado para rcgistrar y organizar la información (Esteval y Reycs, 1998).

La reflexión y sistcmatización de la autoformación permiticron claborar conocimientos, actitudes y comportamientos pensados y vividos no sólo por las profesoras y los profesores que aticnden cl plan de cstudios de prescolar sino, tambićn, por las investigadoras mismas.

La investigación se orientó hacia un marco de

Eil proceso de autoformacion fuc organizado flexibilidad y posibilidades de acción.

El método propuso inicialmentc una cvaluación diagnóstica parara recoger la información y tomar decisiones en cuanto a las neccsidades de alutoformación del personal docentc, para determinar el cstado de presencia del cje transversal sobre desarrollo sostcniblc, cs decir, su coherencia y pertinencia.

Posteriormente se plantcó una autoformación sustentida en un plan de acción (elaborado sobre la base de decisiones de la cvaluación diagnóstica) que sirvió como guía a la autoformación sobrc coduciación ambiental. En cl desalrro. llo de! plan de acción se consideraron situaciones emergentes propias de un proceso cualitativo.

Lil autoformación atendió tanto lass individualidades como la colectividad y sus interrelaciones. Lo subjelivo sc respetó a la vez. que se confrontó con referentes tcóricos, durante procesos reflexivos que llevaron a la construcción de propuestas (compromisos) en los ámbitos de los conocimientos, las actitudes y valores y los comportamientos ambientalmente

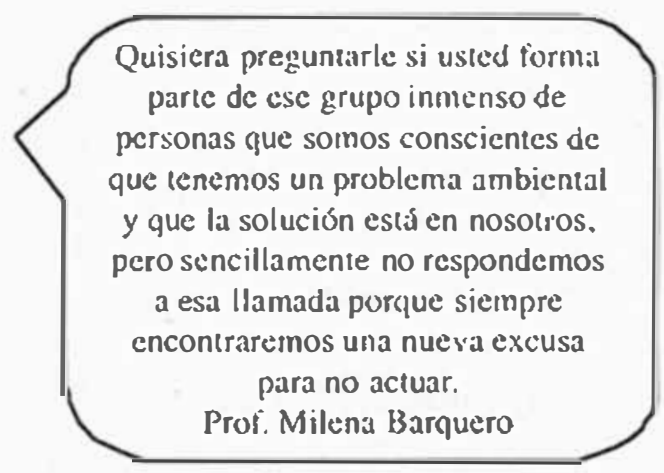
positivos, incluyendo habilidades y destrezals. A su vez los compromisos se realizarron en diferentes áreas de la vida humana: personales y profesionales. 


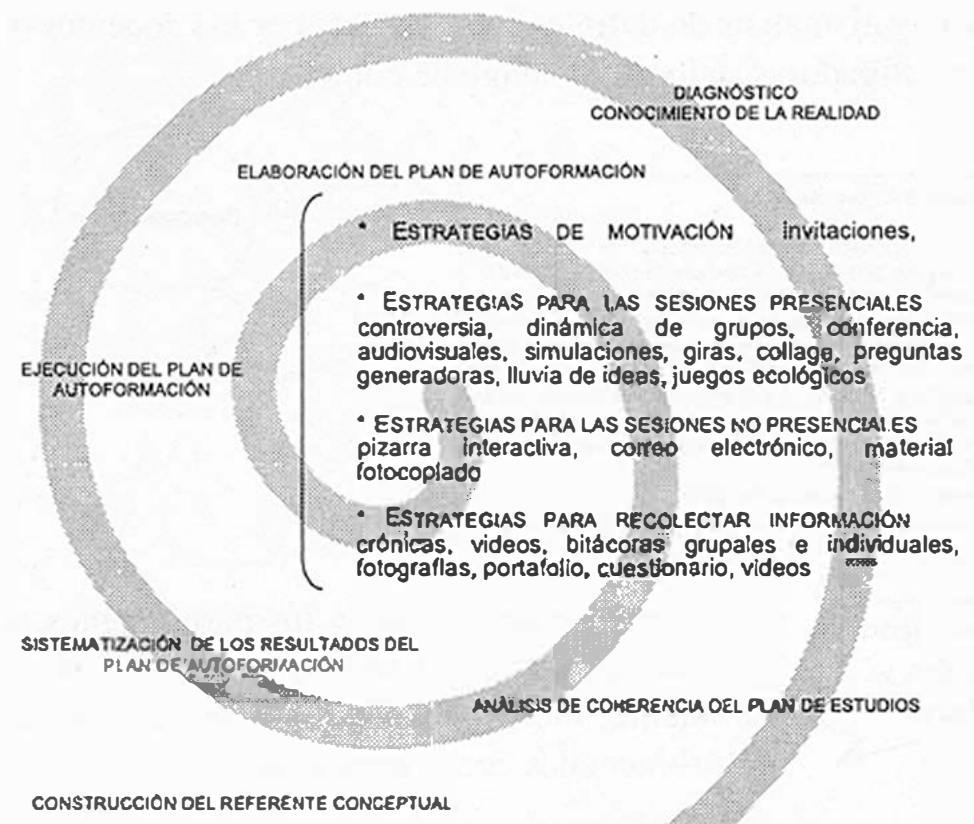

SISTEMATIZACIONOEL PROCESO DE CAMBIO DE LAS INVESTIGADORAS

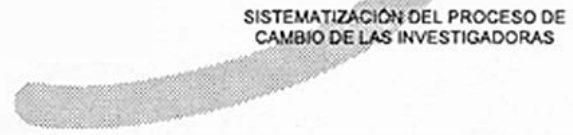

Paralelo al proceso de autoformación vivido por las profesoras y los profesores, las investigadoras desarrollaron un plan de especialización en conocimientos relacionados con diferentes aspectos ambientales $\mathrm{c}$ investigativos.

En términos generales, el siguiente esquema visualiza el proceso de autoformación:

\section{¿Qué generó este proceso de autoformación?}

Los resultados del proceso de autoformación vivenciados con todas las personas participantes pueden valorarse en tres ámbitos principales: conocimientos, actitudes (considerando los valores) y comportamientos (incluyendo habilidades y destrezas).

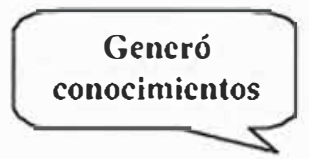

Al inicio del proceso se evidenció una diversidad de conceptos, dados según la experiencia y el conocimiento de cada docente, situación que se convirtió más bien en una fortaleza del grupo, pues se efectuó un intercambio desde diferentes perspectivas en el análisis de un mismo tópico. Sin cmbargo, por otro parte, no había un lenguaje común entre las personas participantes, al referirse a los conceptos de desarrollo sostenible, educación ambiental y dimensión ambiental. De esta mancra, durante el proceso de autoformación, mediante 
la reflexión y el análisis de definiciones creadas por los docentes o propucstas por las investigadoras se logró un lenguaje común.

Eduescion Anotontal.

-Es un proceso transdiscipirrario de farmacion permanonte que so da de manera formal. no fornisl o informal, Impice acciones que promuovon docisiones politicas encaminades a forniar seres humsios en lo conservación del ambiento. propicia l disicgo uns. versal que lova al cambio de actitud y compos. unienios y foments la pez on tes intoraocionos onlse Wos seres vivos y of nxodio ambiente y la capactiad para resolves prostomas y frenslormar y rohabilitar la realidar ambientar".

(Cerdas y Edsovectia, Finio. 2002).
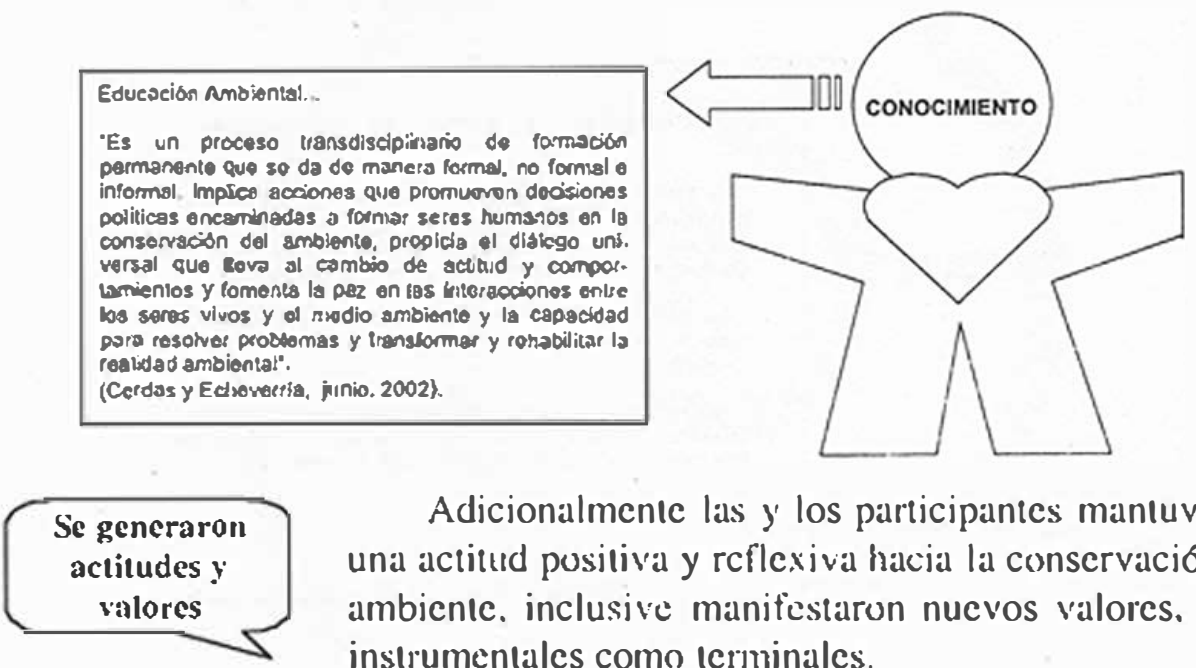

Adicionalmente las $y$ los participantes mantuvicion una actitud positiva y rcflexiva hacia la conservación del ambiente. inclusive manifestaron nuevos valores, lanto instrumentales como terminales.

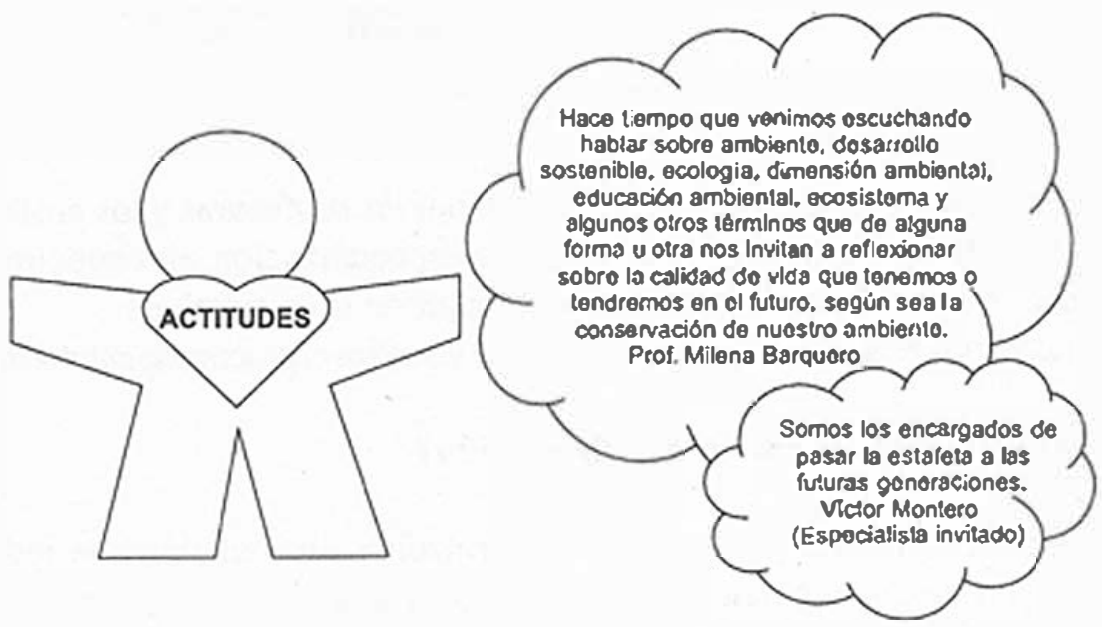

Además se generaron actitudes de intercambio con otras personas, las cualles se expresan como conductas propias de entes multiplicadores. En la propuesla de Cadulo (1985) se evidencia el respelo a sí mismo y a los demás como un valor que trasciende la barrera del deseo por acluar de determinada manera para convertirse en una finalidad de la vida misma.

En cl ámbito educativo se apreció un compromiso para aprovechar espalcios en los cursos tendientes a educar a estudiantes sobre el medio; además se manifestaron descos de ser cjemplo en cuanto a la capacidad de vivir en armonía con la naturalcza. 
Al finalizar el procesi de autoformación se mantuvieron actitudes en las que se destacó la función como entes multiplicadores, por parte de docentes, pues se indicó la intención de efectuar un esfucrzo por involucrar a cstudiantes en un programa que promovicse conocimientos, actitudes y comportamientos en pro de la conservación del ambiente.

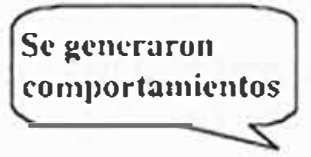

Puede distinguirse la existencia de un comportamiento que permite conocer el ambiente de manera recreativa, en donde se interactúa y disfruta libremente.

A I finalizar la autotormación, las profesoras y los profesores en su totalidad asumicron el compromiso de cambiar sus hábitos de consumo, comportamiento que tiene gran trascendencia pues implica un cambio en su estilo de vida, reflejado no solo en el ámbito profesional sino también en el personal.

También, sc hizo énfasis en cl campo profesional, tanto con las cstudiantes de precscolar como con los niños y niñas en los salones de clase, principalmente con respecto al uso de las "cuatro erres" (Rechazar, Reducir, Reutilizar, Reciclar) como una buena altemativa para reducir la contaminación.

Las y los docentes estaban realizando cambios en las actividades y' apartados de sus programas de curso, o bien,


habían asumido el compromiso de hacerlo a la mayor brevedad, incorporando el cje transversal de desarrollo sostenible en su acción didáctica.

Además demostraron ser concientes de que son parte del ambiente, por lo que algunas se preocuparon por mejorar su entomo inmediato (casa, lugar de (rabajo, edificio de precscolar, UCR).

Por otra parte, en este resultado se encontraron producciones intelectuales donde los y las docentes asumian su papel como entes multiplicadores, a través de medios escritos, en los que se divulgaría la falta de conciencia respecto del detcrioro ambiental. Además reconocicron la necesidad de indagar más en la didáctica ambiental para crear otras altemativas en la acción ceducativa.

Es importante mencionar que utilizaron papel reciclado y reutilizado para escribir o hacer sus producciones. Se puede decir que las habilidades y destrezas desarrolladas por los profesores durante esta actividad comprendicron: 
- Utilización de matcrial reutilizable. por cjemplo, una caja de cartón.

- Creatividad c innovación para utilizar matcriales de desecho o maltcriales que no diñen el ambientc.

- Reflexión c imaginación para descubrir utilidad en malcriales que parecicran inscrvibles.

Al finalizar esic proceso de autoformación. las y los docentes modificaron sus cursos y plantcaron compromisos para incorporar el cje transversal de desalrrollo sosıenible en la acción didáclicia.

Ėl proceso de autoformación generó cambios en las investigadoras.



El cquipo investigador tuvo cambios significalivos a travćs del proceso, como consccuencia de las acciones que realizó en el ámbito personal, profesional y como investigadoris.

Càda una registró csıc proceso a través de bitácoras personalles, grupalcs, y de un auto examen. A continuación, se presental un extracto de este proceso que guarda fidelidad con los ámbitos de conocimientos, valores y actitudes, comportamientos que son un referente durante toda la autoformación.

- El uso de hertamienta tecnológical en cl trabajo cotidiano.

- Utilización de diversas formas de

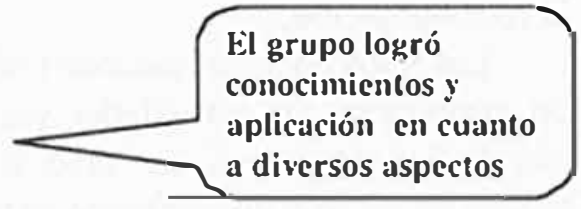
comunicación, oral, corporal y cscrilia.

- Construcción y reconstrucción de referentes conceptuales tiales como: valores ambientales, legislación ambiental, ceducación ambiental, dimensión ambiental, ccología, contaminación ambiental, entes multiplicadores. comunidades de aprendizaije y autoformación.

- Análisis del plan de Estudios de Precscolar.

- Concreción de referentes investigalivos tales como: el enfoçue nalturalista, las premisas ontológicas, hcurísticas, epistemológicas, axiológicas y de la investigación - acción.

- Planificación y sisıcmalización de un proceso investigativo.

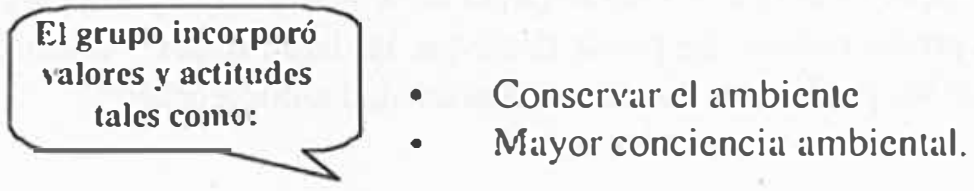


- Amor a las personas y a la Tierra.

- Responsabilidad para asumir el trabajo en equipo

- Ahorrar tiempn, energía, dinero, matcrialcs (vivencia del concepto de desarrollo sostenible y cl valor de autodisciplina).

- Respeto y tolerancia por las diferencias individuales, conviccioncs y creencias de los demás.

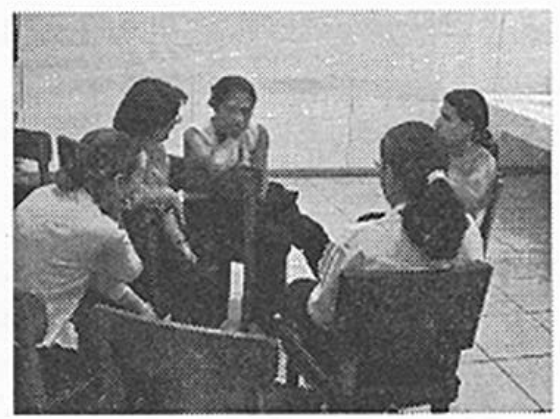

- Perseverancia como una actitud fundamental de la persona que pretende llevar a cabo una investigación-acción.

- Mediación para lograr acucrdos grupales en beneficio del proceso investigativo.

- Solidaridad en diferentes situaciones.

\section{El grupo realizó acciones tales como:}

Trabajo cn equipo, en un ambiente derespeto y tolerancia ante las fortalezas y debilidades de cada integrante.

- Búsqueda de crecimiento integral dentro de los ámbitos cognitivo, emocional y afcctivo.

- Organizar los recursos humanos (docentc, niños, padres, comunidad) y logísticos, para realizar actividades en forma efectiva y eficaz.

De esta mancra y atendiendo al marco de referencia, el grupo investigador logro un desarrollo de actitudes y valores significativos, según se visualiza a continuación:

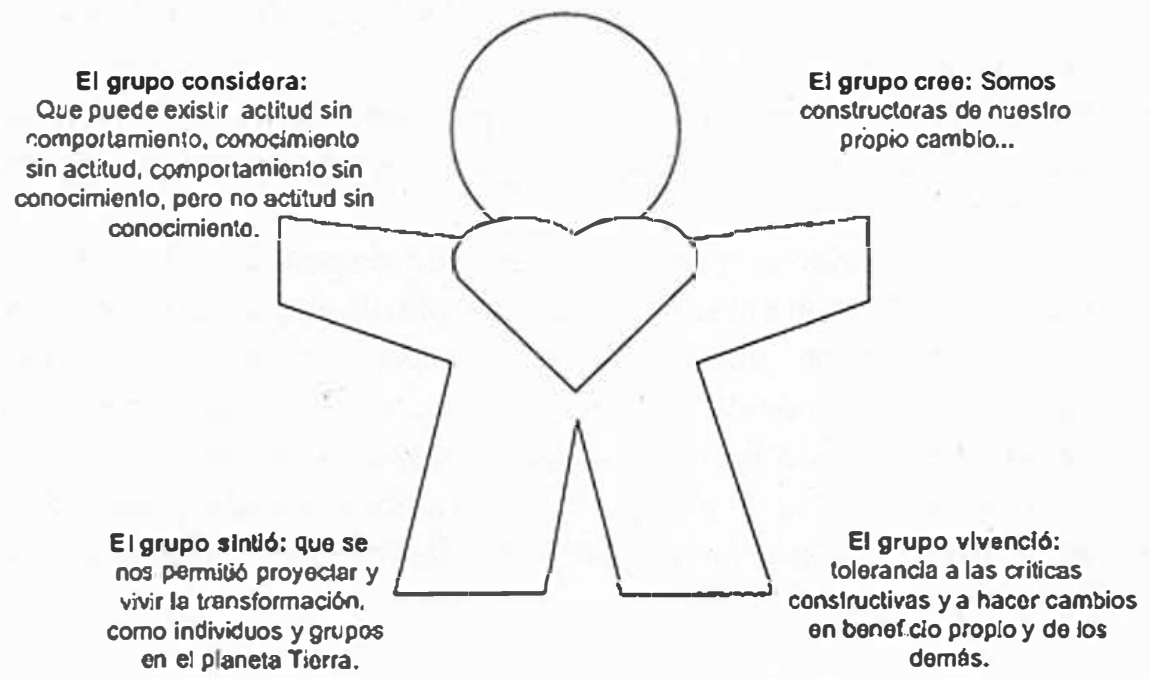




\section{¿Qué se concluye al finalizar el proceso de autoformación?}

Las autoras consideran que el proceso de autoformación fue significativo por cuanto:

1. El proceso de autoformación, hizo posible que las personas participantes alcanzaran un alto nivel de implicación, lo que faciliłó la constancia y su actitud positiva.

2. El nivel de implicación perınitió que los participantes interactuaratn de manera comprometida, cn un marco de comunicación horizontal y transparentc.

3. La autoformación como estrategia metodológica evidenció ser inherente al cnfoque de investigación-acción.

4. La autoformación como estratcgia puede ser aplicada en espacios donde exista una comunidad de aprendizaje, ya sca bajo un marco de investigación o no.

5. El modelo de comportamiento ambiental reflexivo, se convirtió en el "cje transversal" que permeó y brindó sustento tcórico al proccso investigativo.

6. Los profesorcs logratron constituirse en entes multiplicadores al "empodcrarse" de conocimientos, actitudes y valores ambientalcs. Sólo de esta manera fuc factible que rompicran las barreras que impedian la trascendencia hacia un Comportamiento Ambiental Reflexivo.

7. Se generaron reflexiones adicionales, como las siguientes:

- Para lograr un cambio en la forma de trabajar la materia ambiental se debe contemplar la multiplicidad de realidades participantes y visualizarlas como dinámicas.

- Para lograr cambios permanentes y profundos en cl "cmpoderamiento ambiental", es conveniente facilitar cspacios de autoformación que permitan una participación activa.

- La apropiada elección de estrategias que respondan a los intercses y necesidades del contexto, facilita cl desartollo de un plan de autoformación.

- El concepto de entc multiplicador es enriquecido cuando se incorporan las características de compartir, concienciar y contribuir a la formación de las personas que le rodean, es por ello que sc traspasan las barneras individuales para lograr una acción o comportamiento ambientalmente positivo.

- En la investigación-acción la claridad de las relaciones entre sujeto-objeto es vital para su desarrollo 
El proceso sigue...

Ahorabien, siendo quuc los procesos de autoformación son casi inixiabados, al igual que los precesos de investigación-acción, cstc Trabajo Final de Gràduación generó, por parte de su directora, cl desarnollo de una investigaciớn inscrita en el Instituto de Investigaciones para cl Mejorămiento de la Educación Costarricense (IIMEC), cuya meta es dar seguimiento a los propósitos adquiridos por las personas participantes y que se desarrolla para los años 2003 y 2004.

\section{Referencias}

Alvarado, V., Montcro, M.: Rodrígucz, M., Vargas, M.; Vargas, G. (2(X)2). Do). cumentos generados durame el proceso de la investigacion-accionn Ausefonmación sobre Echucacion Ambiental en formadores de docenses de Ledncaciom Presescolar: Scminario de graduación. Escucla de Formación Docentc. Universidad de Cosıa Rica.

Brenes, M. (1992). La imestigacion cualitativa en la educacion latinoamericana. San José: Publicaciones de la Universidad de Costa Rica. Instituto de Investigación y Mcjoramiento Educativo Costanicensc.

Caduto, M. (1985). Guia de Enseñanza de Valores Ambientales. Programa Intcrnacional de Educación Ambicntal UNESCO-PNUMA.

Colás, M. (1994). Investigacionn educativa. Sevilla: Ediciones Alfar, S.A.

Dobles, M. C.; Zúñiga, M., Gaxiáa, J. (1996). Investiggación en educación: procesos, imeracciones, construcciones. San José: Editorial Universidad Estatal a Distancia.

Emmons, K. (1997). Perspectivas en accion ambiental: Reflexion a través de la experiencia práctica. Joumal of Environmental Education. Vol. 29, No. 1,28-33.

Esteva, J., Reycs, J. (1998). Mamual del promotor y educador ambiental para el desarrollo susicentable. México: PNUMA.

Mata, A., Zúñiga, C., Brenes , O., Cartillo, M.; Charpentier, C.; Hernández, L.; Zủñiga, M. (2002). Esstrategias curriculares imnovadoras para la formación inicial de educadores de primaria, en el campo de la lidacacion Ambienal. Comisión Intcruniversitaria de Educación Ambiental (CIEA), CONARE.

Pércz, M. (1990). Investigeación acción en el plano educativo y social. Madrid: Dykinson. 